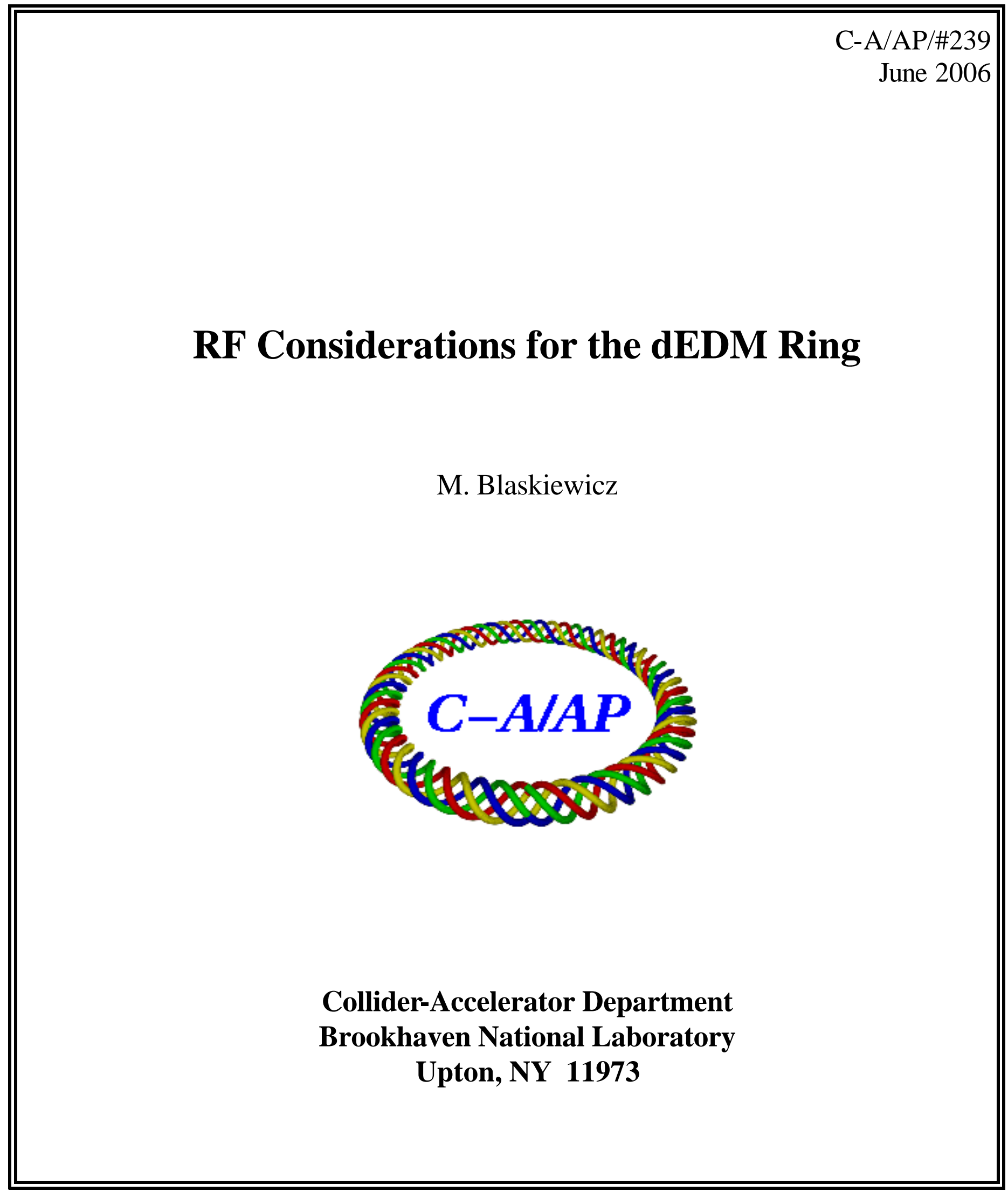




\title{
RF considerations for the dEDM ring
}

\author{
M. Blaskiewicz* \\ $B N L$, Upton NY 11973, USA
}

\begin{abstract}
A storage ring to measure the deuteron electric dipole moment (dEDM) has been proposed [1]. The bunches execute large amplitude coherent synchrotron oscillations in phase lock with coherent spin oscillations leading to a secular change in the spin direction that is proportional to the dEDM. This note addresses relevant $\mathrm{RF}$ issues
\end{abstract}

\section{INTRODUCTION AND THEORY}

Typical parameters for the dEDM ring are shown in Table I. The RF system must bunch the beam and then excite coherent longitudinal oscillations with coherent tune $Q_{c}=\gamma|a|=0.178 \ldots$ Such a large tune implies a superconducting RF system. Since such systems are narrow band the drive for the phase oscillations will be provided by an additional RF cavity. Suppose there are $h$ bunches in the ring. If all the bunches are to oscillate in phase then the frequency of the driving RF could be $f_{d}=\left(h+Q_{c}\right) f_{0}$ or $f_{d}=\left(h-Q_{c}\right) f_{0}$. For a $180^{\circ}$ phase shift between bunches $f_{d}=\left(h / 2 \pm Q_{c}\right) f_{0}$. The relative phase of the coherent longitudinal oscillations must remain phase locked to the coherent spin oscillations over the course of a 20 minute store so a feedback system based on spin measurements is needed. This note outlines a possible system.

\section{GENERAL CONSIDERATIONS}

Let $\theta$ denote machine azimuth, which increases by $2 \pi$ each turn. The RF is confined to a single straight section and the entire system is modeled by a thin lens at $\theta=0$. The intrinsic error associated with this approximation is determined by the fractional velocity modulation, which is significant enough to require additional work but should not influence the underlying principles.

Consider a single particle and let its arrival time at the RF on turn $n$ be $n T_{0}+\tau_{n}$. Let $E_{0}$ be the synchronous energy and $\epsilon_{n}=E_{n}-E_{0}$ be the energy deviation of the particle just before it reaches the RF cavity. The single particle update equations are

$$
\begin{aligned}
\epsilon_{n+1} & =\epsilon_{n}+q V_{R F}\left(n T_{0}+\tau_{n}\right) \\
\tau_{n+1} & =\tau_{n}+\eta T_{0} \frac{\epsilon_{n+1}}{\beta^{2} E_{0}}
\end{aligned}
$$

where $\eta=\alpha_{p}-1 / \gamma^{2}$ is the frequency slip factor and $V_{R F}(t)$ is the total RF voltage at time $t$. The voltage consists of the primary RF with amplitude $V_{1}$ and frequency $h f_{0}$ and a driving term $V_{d}$ at $f_{0}\left(h_{d}+Q_{c}\right)$, where $h_{d}$ is an integer.

TABLE I: dEDM ring parameters

\begin{tabular}{lc}
\hline \hline parameter & value \\
\hline circumference & $C=25 \mathrm{~m}$ \\
Lorentz factor & $\gamma=1.25$ \\
revolution period and frequency & $T_{0}=138.98 \mathrm{~ns}=1 / f_{0}$ \\
momentum compaction factor & $\alpha_{p}=1$ \\
anomolous magnetic moment & $a=-0.142988$ \\
number of ions in the ring & $N_{t} \sim 10^{12}$ \\
nuclear mass and charge & $M=1.876 \mathrm{GeV} / \mathrm{c}^{2}, \mathrm{Z}=1$ \\
\hline \hline
\end{tabular}

*Electronic address: blaskiewicz@bnl.gov 
Then

$$
\begin{aligned}
V_{R F}\left(n T_{0}+\tau_{n}\right) & =V_{1} \sin \left(h \omega_{0} \tau_{n}\right)+V_{d} \sin \left(\omega_{0}\left[h_{d}+Q_{c}\right] \tau_{n}+2 \pi n Q_{c}\right) \\
& \left.\approx V_{1} \sin \left(h \omega_{0} \tau_{n}\right)+V_{d} \sin \left(2 \pi n Q_{c}+\omega_{0}\left[h_{d}+Q_{c}\right] \tau_{0}\right)\right)
\end{aligned}
$$

where $\omega_{0}=2 \pi f_{0}, \tau_{0}$ corresponds to the stable fixed phase for the particle, and the approximation of equation (4) will be used only for analytic work. While equations (1), (2) and (3) are used in simulations the author prefers the continuum limit to develop understanding. Toward this end let $\theta$ be the time-like variable, and $\phi=h \omega_{0} \tau$ be the position variable so that

$$
\frac{d^{2} \phi}{d \theta^{2}}=-Q_{s}^{2}\left\{\sin \phi+\frac{V_{d}}{V_{1}} \sin \left(Q_{c} \theta\right)\right\}
$$

where the stable fixed point is at $\phi=0$, the phase shift $\omega_{0}\left[h_{d}+Q_{c}\right] \tau_{0}$ has been absorbed in $\theta$ and the small amplitude synchrotron tune is

$$
Q_{s}=\sqrt{\frac{2 \pi q|\eta| h V_{1}}{\beta^{2} E_{0}}} .
$$

For $r \equiv V_{d} / V_{1} \ll 1, \sin \phi+r \sin \left(Q_{s} \theta\right) \approx \sin \left(\phi+r \sin \left[Q_{s} \theta\right]\right)$ and the dynamics of the two harmonic system are similar to the dynamics of a phase modulated single harmonic system. The latter has been studied in detail [2]. Setting the momentum coordinate to

$$
p=\frac{1}{Q_{s}} \frac{d \phi}{d \theta}
$$

equation (5) can be derived from the hamiltonian

$$
H(\phi, p, \theta)=Q_{s} p^{2} / 2+Q_{s}\left\{1-\cos \phi+\phi r \sin \left(Q_{c} \theta\right)\right\} .
$$

Consider a canonical transformation of Goldstein's [3] first type with generator

$$
F_{1}=\frac{\phi^{2}}{2} \cot \left(\psi+Q_{c} \theta\right)
$$

where $\psi$ is the new position coordinate. The old momentum coordinate is

$$
p=\frac{\partial F_{1}}{\partial \phi}=\phi \cot \left(\psi+Q_{c} \theta\right),
$$

and the new momentum coordinate is

$$
J=-\frac{\partial F_{1}}{\partial \psi}=\frac{\phi^{2}}{2 \sin ^{2}\left(\psi+Q_{c} \theta\right)},
$$

so that

$$
\phi=\sqrt{2 J} \sin \left(\psi+Q_{c} \theta\right), \quad p=\sqrt{2 J} \cos \left(\psi+Q_{c} \theta\right) .
$$

The new hamiltonian is given by

$$
\begin{aligned}
H_{2}(\psi, J, \theta) & =H+\frac{\partial F_{1}}{\partial \theta} \\
& \approx\left(Q_{s}-Q_{c}\right) J-Q_{s} J^{2} / 16+Q_{s} \frac{r}{4} \sqrt{2 J} \cos \psi
\end{aligned}
$$

where equation (8) is obtained by dropping terms with frequency $2 Q_{s}$ and higher and by dropping terms $O\left(J^{4}\right)$. A final canonical transformation with position variable $x=\sqrt{2 J} \cos \psi$ and momentum $v=-\sqrt{2 J} \sin \psi$ yields the final hamiltonian

$$
K(x, v)=\frac{Q_{s}-Q_{c}}{2}\left(x^{2}+v^{2}\right)-\frac{Q_{s}}{64}\left(x^{2}+v^{2}\right)^{2}+Q_{s} \frac{r}{4} x
$$




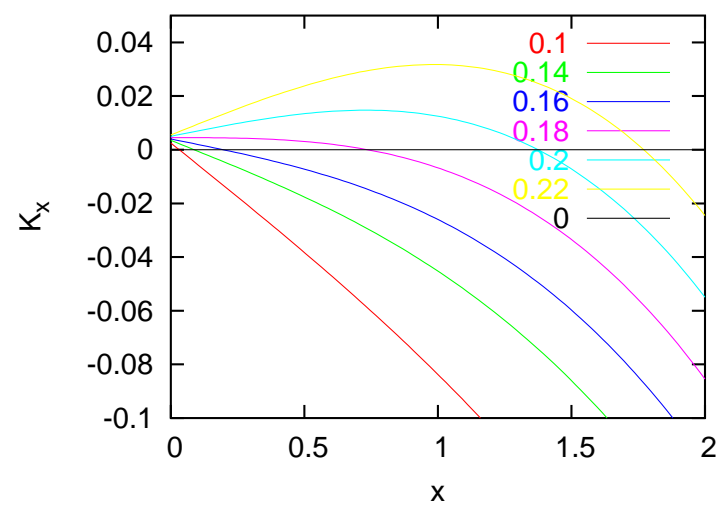

FIG. 1: $K_{x}(x, p=0)$ with $r=0.10, Q_{c}=0.18$, and various values of $Q_{s}$. For $Q_{s}=0.10$ there is one stable fixed point near $x=0$. As $Q_{s}$ grows this fixed point smoothly migrates to large values of $x$.

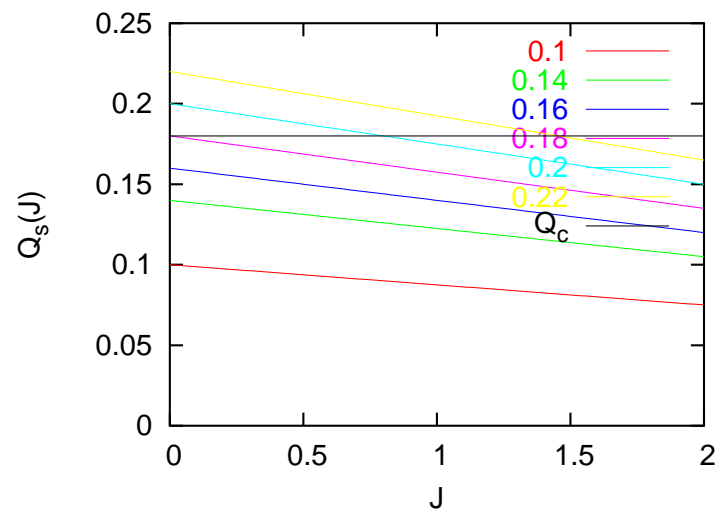

FIG. 2: Synchrotron tune versus action for the cases of Figure 1. The drive tune is $Q_{c}$ (black line) and the beam evolves from small tunes (red curve) to large tunes.

The fixed points of $K$ satisfy $\partial K / \partial x \equiv K_{x}=K_{y}=0$, and the fixed point is stable if $K_{x x} K_{y y}>0$. Figure 1 shows a plot of $K_{x}$ for various values of $Q_{s}$ with $r$ and $Q_{c}$ fixed. By studying this figure a fully adiabatic technique for producing large amplitude oscillations can be obtained. First consider the red curve with $Q_{s}=0.1$. This curve crosses zero only once, near $x=0.05$. In the lab frame all the particles will oscillate in phase with this small amplitude. Now consider what happens as the main RF voltage is slowly raised and $Q_{s}$ increases. The stable fixed point smoothly moves to larger values of $x$ and, if the beam emittance is small enough, all the particles will remain near the stable fixed point. In the lab frame this corresponds to a smooth increase in the synchrotron oscillation amplitude. For a somewhat more intuitive approach consider Figure 2.

The initial state in Figure 2 corresponds to the red curve where all the particles have a tune less than the drive tune. As the main RF voltage is raised the tune distribution rises toward $Q_{c}$. Particles at small amplitude are excited first, since they have the largest $Q_{s}$, and their amplitude increases. By the time the tune distribution at $J=0$ reaches $Q_{c}$, no particles have $J=0$. As the crossing point, defined by $Q_{s}(J)=Q_{c}$, moves to larger $J$ the entire distribution of particles is continually pushed to the right (large $J$ side) of the crossing point. The next section presents some initial computer simulations used to flesh out these ideas.

\section{SIMULATIONS}

Simulations are based on equations (1) and (2). The RF voltage has 3 components,

$$
V_{R F}(t)=V_{1}(t) \sin \left(h_{1} \omega_{0} t\right)+V_{2}(t) \sin \left(2 h_{1} \omega_{0} t\right)+V_{d}(t) \sin \left(\left[h_{d}+Q_{c}\right] \omega_{0} t\right),
$$

where $h_{1}$ is the primary harmonic number, $h_{d}$ is the coherent drive harmonic, and the three voltage amplitudes $V_{1}, V_{2}, V_{d}$ vary slowly. The second harmonic voltage $V_{2}$ has been included to increase control over the tune variation 


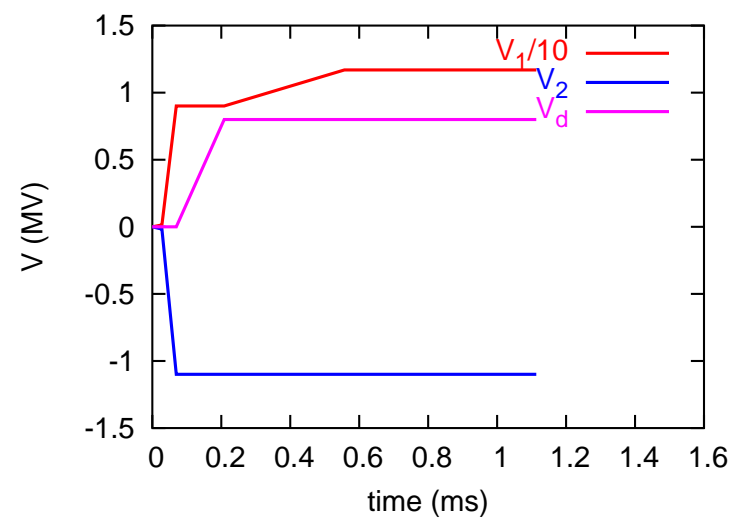

FIG. 3: Voltage amplitudes versus time with $h_{1}=50$ and $h_{d}+Q_{c}=25.1788$.

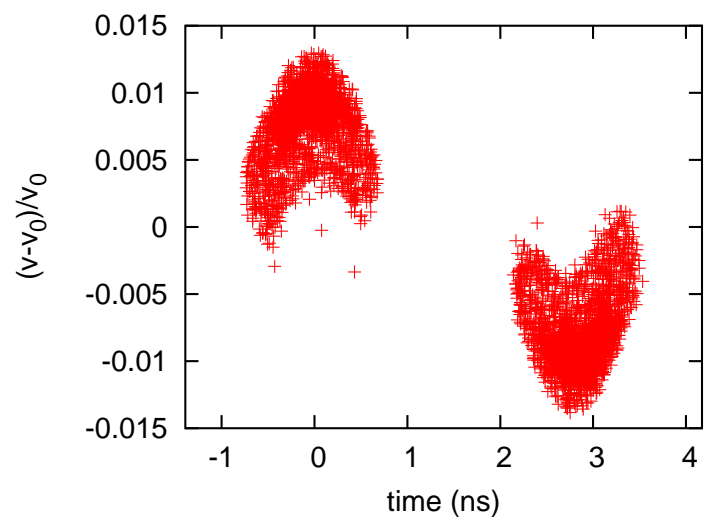

FIG. 4: Phase space plots on a single turn for two adjacent bunches.

with amplitude. Assume that the AGS injects a single bunch at $t=0$ which subsequently debunches in the dEDM ring. Typical lots of the voltage amplitudes versus time are shown in Figure 3. Note that the millisecond time axis is not to be taken literally. With our adiabatic technique one could stretch out the time axis to several seconds without changing the results. A plot of two adjacent bunches near the end of the simulation are shown in Figure 2. Note that the primary RF frequency sets the spacing between bunches. The 3 nanosecond bunch spacing corresponds to a $360 \mathrm{MHz}$ fundamental $\mathrm{RF}$ which is the lower range of available klystron frequencies.

\section{Acknowledgements}

Thanks to Yuri Orlov, Bill Morse, and Yannis Semertzidis for useful discussions and encouragement.

[1] Y. Orlov, W. Morse, Y. Semertzidis Phys. Rev. Lett. 96, 214802 (2006).

[2] H. Huang et. al. Phys Rev E, 48 \# 6, p4678, (1993).

[3] H. Goldstein, Classical Mechanics Addison-Wesley, 1981. 